\title{
Twitter as a Tool to Spread Communication Regarding Genitourinary Cancers During the COVID-19 Pandemic
}

\author{
Sabrina Salgia ${ }^{\mathrm{a}}$, Nicholas Salgia ${ }^{\mathrm{a}}$, Sweta Prajapati ${ }^{\mathrm{a}}$, Ishaan Seghal ${ }^{\mathrm{a}}$, Frank Bautista ${ }^{\mathrm{a}}$, Nora Ruel $^{\mathrm{b}}$, \\ Meghan Salgia $^{a}$, Deborah A. Salgia ${ }^{c}$, Ravi Salgia ${ }^{a}$ and Sumanta K. Pal ${ }^{a, *}$ \\ ${ }^{a}$ Department of Medical Oncology \& Experimental Therapeutics, City of Hope Comprehensive Cancer Center, \\ Duarte, CA, USA \\ ${ }^{\mathrm{b}}$ Biostatistics and Mathematical Modeling Core, City of Hope Comprehensive Cancer Center, Duarte, CA, USA \\ ${ }^{\mathrm{c}}$ Department of Supportive Care Medicine, City of Hope Comprehensive Cancer Center, Duarte, CA, USA
}

Received 18 February 2021

Accepted 9 April 2021

Pre-press 11 May 2021

Published 16 June 2021

\begin{abstract}
.
OBJECTIVES: To better characterize the relay of information about prostate, kidney, and bladder cancer on Twitter in relation to the COVID-19 pandemic.

MATERIALS AND METHODS: Tweets containing the joint hashtags “\#COVID-19” and either "\#bladder cancer", “\#kidney cancer", or "\#prostate cancer" were identified on the Twitter platform from January 1, 2020 to July 30, 2020. The Twitter handle responsible for each tweet was categorized as an Academic, Medical Education, Patient Advocacy Groups/NonProfits, Pharmaceutical, or Other entity based on content domain. Descriptive statistics were used to summarize data on Twitter handle characteristics stratified by disease category (bladder, kidney, and prostate). Median/interquartile range and percentages were used to summarize continuous and categorical data, respectively. Number of tweets containing the relevant joint hashtags were tracked over time in relation to the cumulative United States case count of COVID-19.

RESULTS: The content of 730 total tweets containing the joint hashtags "COVID-19" and either "\#bladder cancer" (138 tweets), "\#kidney cancer" (137 tweets), or "\#prostate cancer" (455 tweets) from January 1, 2020 to July 31, 2020 were analyzed. We identified 326 unique Twitter handles across all disease states (62 bladder, 47 kidney, and 217 prostate-related). Academic Twitter handles accounted for the greatest number of tweets containing the joint hashtags (31\%). Temporal tracking of tweets with regard to monthly U.S. COVID cases revealed that communication surged in March of 2020 and peaked in April for both bladder and kidney cancer, whereas related prostate cancer Twitter communication peaked in May of 2020.

CONCLUSIONS: As COVID-19 case counts rose in the United States initially, so too did communication surrounding COVID-19 and genitourinary cancers on Twitter. Many of these conversations were driven by academically-associated Twitter accounts.
\end{abstract}

Keywords: Bladder cancer, COVID-19, kidney cancer, prostate cancer, Twitter

\footnotetext{
${ }^{*}$ Correspondence to: Sumanta K. Pal, Department of Medical Oncology \& Experimental Therapeutics, 1500 East Duarte Road,
} 


\section{INTRODUCTION}

Social media is currently used as a global tool to communicate information, opinions, and updates. As of $2019,72 \%$ of U.S. adults used at least one social media platform to relay information, up from only $5 \%$ in 2005 [1]. Since its creation in 2006, Twitter has served as a platform that unites mass communication of both the social and professional sectors. Each day 500 million tweets are sent with an active 152 million Twitter users daily [2].

Twitter usage in the domain of oncology has followed similar trends to that of the public [3]. The growing usage of social media among patients, professionals, and organizations has opened up new areas of research into cancer communication. Previous studies have found that cancer-related content on Twitter tends to revolve around delivering information related to care and support [4]. There is also a growing interest for oncology providers to use Twitter as a platform to network with colleagues and health care organizations [5]. Associated oncological entities, including pharmaceutical companies, nonprofit organizations, and patient advocate groups have established a communication platform on Twitter, each with their unique goals and vision [6-8].

While Twitter continues to grow as a communication tool in oncology, the onset of the COVID-19 pandemic has shifted the focus of communications for healthcare entities and public health officials. The World Health Organization (WHO) and other federal agencies have continuously published COVID-19 content through their Twitter accounts, providing credible sources of information during this crisis [9]. Characterization of this shift towards COVID19 messaging remains unaddressed among Twitter users dedicated to disseminating cancer-related information.

The intersection of COVID-19 and cancer poses a direct threat to many patients. Cancer patients are often immunocompromised, older ( $>60$ years), and maintain one or more significant comorbidities; these factors put those living with cancer at greater risk for COVID-19 morbidity and mortality. The COVID-19 and Cancer Consortium (CCC19) study reported that patients diagnosed with both COVID-19 and cancer were at greater risk of mortality and severe illness secondary to infection [10-13]. A substantial portion $(21 \%)$ of subjects in the CCC19 study had specified genitourinary malignancies. Given these increased risks and the evolution of COVID-19 discussions on social media, we sought to characterize the Twitter communications of both COVID-19 and genitourinary cancers during the COVID-19 pandemic.

\section{MATERIALS AND METHODS}

\section{Study participants}

Three separate searches of joint hashtags were conducted on Twitter messages (tweets) dated from January 1, 2020 until July 31, 2020: “\#COVID-19 and \#kidney cancer", "\#COVID-19 and \#bladder cancer", and "\#COVID-19 and \#prostate cancer". The associated user handle for each identified tweet was categorized as (1) Academic, (2) Medical Education, (3) Patient Advocacy Groups/Non-Profits, (4) Pharmaceutical, or (5) Other. Categorization of each handle was performed based on the user's profile or additional searches.

The total number of tweets were recorded for the joint hashtag searches pertaining to kidney, bladder, and prostate cancer. Additional variables were collected including date the tweet was created, tweet content, number of comments on the tweet, number of likes on the tweet, number of retweets of the original tweet posted, and if the tweet was an original tweet or a retweet. Descriptive variables of the users were also collected: the username of the Twitter handle, the joined date of the Twitter handle, number of following, number of followers, and total number of tweets posted. These variables were quantified using median inter-quartile ranges.

The number of reported new monthly COVID-19 cases in the United States from January 1, 2020 to July 31, 2020 were tracked in congruence $[14,15]$. Comparison of new COVID-19 cases and number of tweets containing the joint hashtag terms were compared for each month from January to July 2020.

\section{Statistical analysis}

Descriptive statistics was used to summarize data on Twitter handler characteristics stratified by disease category (bladder, kidney, prostate cancer). Median and interquartile range, frequencies, and percentages were used to summarize continuous and categorical data, respectively. Log transformations were done for number of followers, number followed, and number of tweets to reduce skewness in distribution and allow for consistency and improved interpretation of the results. Spearman ranked correlation coefficient was calculated to show association between all items related to the Twitter handle and tweeter item 
characteristics and metrics. Heat maps were used for graphic display of these results. The Wilcoxon ranksum and chi-square or Fisher's exact tests were used to compare continuous and discrete data distributions, respectively, between strata of both Twitter handlers and tweets.

\section{RESULTS}

A total of 730 tweets that contained the joint hashtags of "\#COVID-19" and either "\#bladder cancer" $(n=138)$, "\#kidney cancer" $(n=137)$, or "\#prostate cancer" $(n=455)$ were collected from January 1 , 2020 to July 31,2020 . The plurality (31\%) of tweets across all groups were sent by academic entities. Pharmaceutical companies disseminated the least number of tweets that included said joint hashtags (2.9\%). Table 1 provides full characteristics of Twitter-specific variables in relation to tweets using the joint hashtags. There were a total number 326 unique Twitter handles responsible for these tweets, of which 127 (39\%) were classified as academic entities. Academic accounts received more likes and retweets per tweet across all three cohorts.

Heat maps with Spearman correlations were constructed for each genitourinary cancer: bladder cancer, kidney cancer, and prostate cancer. Figure 1 depicts heat maps with canonical correlations of Twitter variables in the context of genitourinary cancers and COVID-19 tweets. Expected correlations were observed between variables linked to experience and recognition on the Twitter platform, including time on platform, number of followers/following, and number of tweets. In addition, expected correlations between likes, retweets and volume of comments were seen. In bladder and kidney cancer, there was a higher correlation between experiential variables (time on platform/number of followers) and popularity of individual tweets (number of likes/retweets) relative to prostate cancer.

Figure 2 outlines temporal trends in COVID-19 cases alongside the number of tweets related to genitourinary cancers. Although initially the rise in COVID-19 incidence was paralleled by an increase in related communication on Twitter, the same rise was not seen with the "second wave" of COVID-19 in July of 2020. The volume of tweets related to prostate, kidney and bladder cancer moved in parallel throughout this period of time, although overall, the volume of tweets pertaining to prostate cancer far exceeded those for kidney and bladder cancer.

\section{DISCUSSION}

This study looked to investigate the temporal trends of Twitter communications involving both the COVID-19 pandemic and genitourinary cancers from January 1 to July 31, 2020. Ultimately, we demonstrated that communication surged in March of 2020 and peaked in April for both bladder and kidney cancer, whereas related prostate cancer communication peaked in May of 2020. Thus, as the initial wave of COVID-19 case counts rose in the United States, so too did communication surrounding COVID-19 and genitourinary cancers on the Twitter platform.

Table 1

Comparison of genitourinary cancers, Twitter categorization, and associated variables with data represented by $\mathrm{N}$ or Median (IQR)

\begin{tabular}{|c|c|c|c|c|c|c|}
\hline & Academic & $\begin{array}{l}\text { Medical } \\
\text { Education }\end{array}$ & $\begin{array}{c}\text { Patient } \\
\text { Advocacy/ } \\
\text { Non-Profit }\end{array}$ & Pharmaceutical & Other & $P$-value \\
\hline \multicolumn{7}{|l|}{ Bladder Tweets ( $n=138$ tweets) } \\
\hline Number of tweets & 56 & 38 & 28 & 1 & 15 & \\
\hline Number of unique Twitter handles & 26 & 16 & 8 & 1 & 11 & \\
\hline Number of Likes per tweet & $4(2-10.5)$ & $3(1-7)$ & $2(1-5)$ & $3(3-3)$ & $1(0-4)$ & 0.05 \\
\hline Number of Retweets per tweet & $2(0-3.5)$ & $1(0-3)$ & $1(0-3)$ & $1(1-1)$ & $0(0-1)$ & 0.17 \\
\hline \multicolumn{7}{|l|}{ Kidney Tweets ( $n=137$ tweets) } \\
\hline Number of Tweets & 38 & 11 & 61 & 1 & 26 & \\
\hline Number of unique Twitter handles & 26 & 3 & 14 & 1 & 3 & \\
\hline Number of Likes per tweet & $6.5(3-12)$ & $4(1-6)$ & $3(2-6)$ & $0(0-0)$ & $0(0-0)$ & $<0.0001$ \\
\hline Number of Retweets per tweet & $3(0-7)$ & $2(0-4)$ & $2(1-3)$ & $0(0-0)$ & $0(0-0)$ & $<0.0001$ \\
\hline \multicolumn{7}{|l|}{ Prostate Tweets ( $n=455$ tweets) } \\
\hline Number of Tweets & 134 & 77 & 183 & 19 & 85 & \\
\hline Number of unique Twitter handles & 75 & 51 & 49 & 12 & 30 & \\
\hline Number of Likes per tweet & $2(0-5)$ & $5(1-15)$ & $2(0-4)$ & $1(0-2)$ & $1(0-5.5)$ & $<0.0001$ \\
\hline Number of Retweets per tweet & $1(0-3)$ & $1(0-5)$ & $1(0-3)$ & $0(0-1)$ & $0(0-2)$ & 0.04 \\
\hline
\end{tabular}


(a)
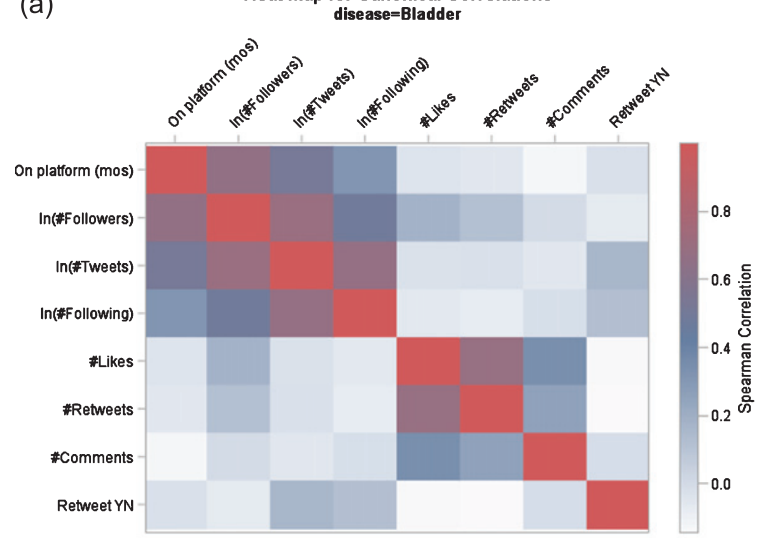

(b)

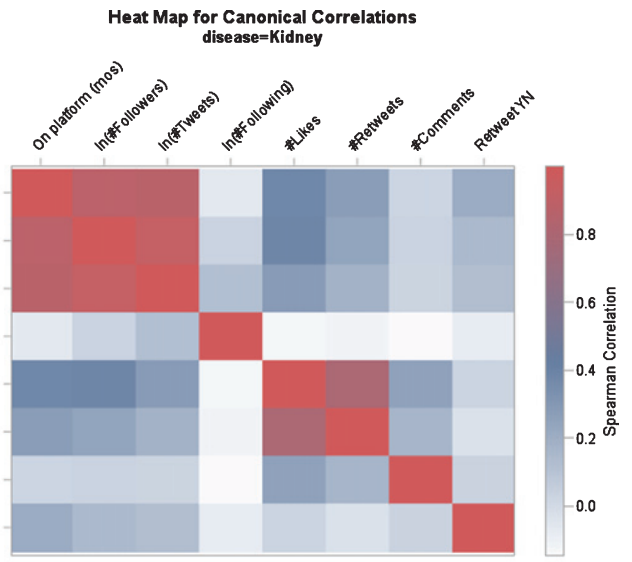

(c)

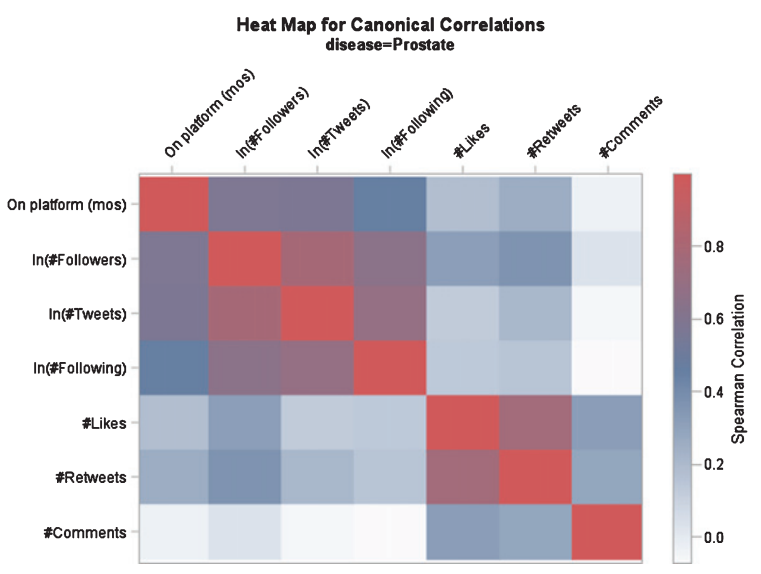

Fig. 1. Spearman correlation heat map for Twitter variable relationships within (a) bladder cancer (b) kidney cancer and (c) prostate cancer cohorts.

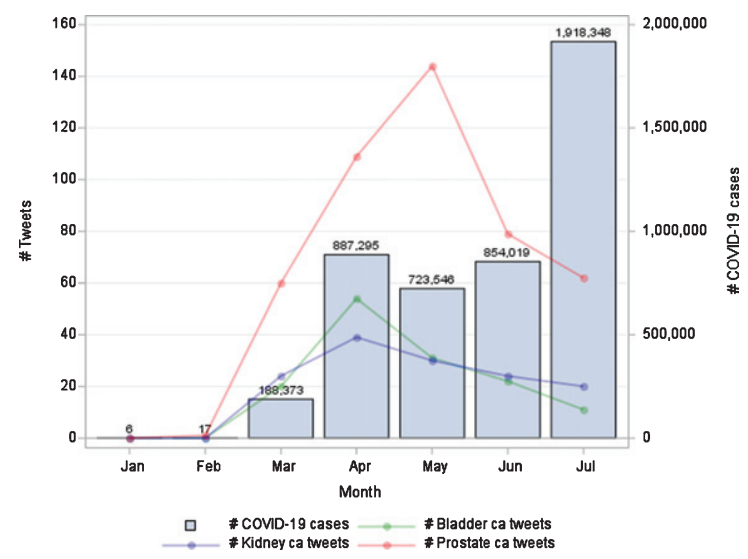

Fig. 2. Temporal tracking of newly diagnosed U.S. COVID-19 cases and specified genitourinary related tweets from January through July of 2020.

Multiple studies have recently reported that social media platforms including Twitter have been inundated with medical misinformation and unverifiable content relating to the COVID-19 pandemic, which is being spread at an alarming fast rate on these social platforms [16-18]. Our findings that a plurality of tweets $(31 \%)$ were from trusted academic organizations - entities that tend to share publications, research-related news, and policy announcements - suggests that genitourinary cancer information is accurately being conveyed to the social media users even in light of the current misinformation trends [19].

This study represents the first effort to investigate the relationship between monthly COVID-19 cases in the U.S. and the associated temporal changes in Twitter communication surrounding genitourinary cancers and COVID-19, of which prostate cancer was the most discussed. Several factors could have contributed to this communication. In 2020, there will be an estimated 191,930 new prostate cancers, 73,750 new kidney cancer cases, and 81,400 new bladder cancer cases diagnosed [6]. This high prevalence of new prostate cancer cases in addition to those living with prostate cancer may contribute 
to the increased communication relative to other genitourinary cancers. Patient advocacy groups/nonprofit organizations have also been reported to utilize Twitter to spread one-way messages surrounding prostate cancer and COVID-19 [20]. Our findings support these conclusions, as $40.2 \%$ of total prostate cancer tweets were from patient advocacy groups and non-profit organizations.

The higher volume of prostate cancer tweets pertaining to COVID-19 could also be driven by key biological insights garnered during the timeframe assessed. Several publications during this timeframe suggested that the TMPRSS2 gene and its protein product play a role both in prostate cancer development and in COVID-19 host infection. TMPRSS2 overexpression and gene fusion with $E R G$ at chromosome $21 \mathrm{q} 22.3$ are present in up to $80 \%$ of prostate cancers and result in upregulation of the ETS transcription factor. ETS plays a critical role in cell differentiation, proliferation, and inflammation fundamental to oncogenesis [21-23]. Additionally, recent evidence suggests that SARS-CoV-2 makes its way into the cell via the ACE2 receptor in synergy with the host's TMPRSS2. The activity of TMPRSS2 is now considered the only critical factor in assisting cell entry of the SARS-CoV-2 viral pathogen [23]. This common reliance on TMPRSS2 may pose as an additional consideration for observed trends of increased discussion surrounding prostate cancer and COVID-19.

Indeed, there are efforts to harmonize medical communication pertaining to cancer and COVID-19. In March of 2020, the COVID-19 and Cancer Consortium (CCC19) was established following grassroots efforts on Twitter to better address the intersection of these two malignancies [12]. To date, there are over 50 cancer centers, universities, and research institutes across the United States actively participating in the CCC19. The CCC19 continues to use Twitter as a platform to relay information, data, and new clinical and research findings in hopes to bridge the knowledge gap in cancer care caused by the COVID-19 pandemic. Twitter serves as a useful platform for collection and dissemination of COVID-19 and cancer related information, as evidenced by the CCC19 and our findings. It is thought that Twitter and other social media platforms may represent a great equalizer in medical access - providing expertise and up-to-date research findings to the public without financial or professional barriers.

This study had several limitations. Tweets related to the COVID-19 pandemic and genitourinary cancers were analyzed from a timepoint of January to July 2020. However, the COVID-19 pandemic has continued to evolve and worsen in the time beyond. By the end of July, the US had seen approximately 3 million positive cases whereas at the end of November 2020, cumulative cases have surpassed 12 million and resulted in over 250,000 deaths [24]. Further analysis to assess the trends of COVID-19 and genitourinary cancers beyond July 2020 may provide a better understanding of changes in Twitter discussions during each spike in cases. Additionally, no specific content analysis was performed on the tweets identified - therefore limiting the extent of our understanding of the topics discussed within the broader classifications of COVID-19 and each genitourinary cancer.

The results reported herein provide novel insights into discussions surrounding the ongoing COVID19 pandemic and genitourinary cancers on Twitter. As COVID-19 cases rose in the United States during the first wave of infection, so too did tweets related to COVID-19 and genitourinary cancers. However, these trends did not continue into the second wave and beyond. As the public appears to rely heavily on social media as an outlet for COVID-19 related news, it will be critical that academicians remain engaged on Twitter and continue to convey trustworthy, evidence-based information on this platform.

\section{ACKNOWLEDGMENTS}

The authors have no acknowledgments.

\section{FUNDING}

Grant “P30 CA033572-33” to Ravi Salgia.

\section{AUTHOR CONTRIBUTIONS}

Sabrina Salgia - conception, editing, writing manuscript, data interpretation; Nicholas Salgia conception, editing, writing manuscript, data interpretation; Sweta Prajapati - data collection; Ishaan Seghal - data collection; Frank Bautista - data collection; Nora Ruel - statistician, data interpretation, editing, writing manuscript; Meghan Salgia - editor; Deborah A. Salgia - editor; Ravi Salgia - editor; Sumanta K. Pal - conception, editing, writing manuscript, data interpretation. 


\section{CONFLICTS OF INTEREST}

Sabrina Salgia, Nicholas Salgia, Sweta Prajapati, Ishaan Seghal, Frank Bautista, Nora Ruel, Meghan Salgia, and Deborah A. Salgia have no conflicts of interest that might be relevant to the contents of this manuscript. Ravi Salgia, MD, PhD: Consulting or Advisory Role: Janssen, AstraZeneca, Novartis, Merck. Sumanta K. Pal, MD: Honoraria: Novartis, Medivation, Astellas Pharma; Consulting or Advisory Role: Pfizer, Novartis, Aveo, Myriad; Pharmaceuticals, Genentech, Exelixis, Bristol-Myers Squibb, Astellas Pharma; Research Funding: Medivation.

\section{REFERENCES}

[1] NW 1615 L. St, Suite 800Washington, Inquiries D 20036USA202-419-4300 | M-857-8562 | F-419-4372 | M. Demographics of Social Media Users and Adoption in the United States. Pew Research Center: Internet, Science \& Tech n.d. https://www.pewresearch.org/internet/factsheet/social-media/ (accessed November 23, 2020).

[2] Twitter by the Numbers (2020): Stats, Demographics \& Fun Facts n.d. https://www.omnicoreagency.com/twitterstatistics/ (accessed November 23, 2020).

[3] Adilman R, Rajmohan Y, Brooks E, Urgoiti GR, Chung C, Hammad N, et al. ReCAP: Social Media Use Among Physicians and Trainees: Results of a National Medical Oncology Physician Survey. JOP 2015;12:79-80. https:// doi.org/10.1200/JOP.2015.006429.

[4] Sedrak MS, Salgia MM, Decat Bergerot C, AshingGiwa K, Cotta BN, Adashek JJ, et al. Examining Public Communication About Kidney Cancer on Twitter. JCO Clinical Cancer Informatics 2019:1-6. https://doi. org/10.1200/CCI.18.00088.

[5] Sedrak MS, Dizon DS, Anderson PF, Fisch MJ, Graham DL, Katz MS, et al. The emerging role of professional social media use in oncology. Future Oncology 2017;13:1281-5. https://doi.org/10.2217/fon-2017-0161.

[6] Pemmaraju N, Thompson MA, Qazilbash M. Diseasespecific hashtags and the creation of Twitter medical communities in hematology and oncology. Seminars in Hematology 2017;54:189-92. https://doi.org/10.1053/j.sem inhematol.2017.08.004.

[7] Murthy D, Eldredge M. Who tweets about cancer? An analysis of cancer-related tweets in the USA. DIGITAL HEALTH 2016;2:2055207616657670. https:// doi.org/10.1177/2055207616657670.

[8] Koskan A, Klasko L, Davis SN, Gwede CK, Wells KJ, Kumar A, et al. Use and Taxonomy of Social Media in Cancer-Related Research: A Systematic Review. Am J Public Health 2014;104:e20-37. https://doi.org/10. 2105/AJPH.2014.301980.

[9] Wang Y, Hao H, Platt LS. Examining risk and crisis communications of government agencies and stakeholders during early-stages of COVID-19 on Twitter. Computers in Human Behavior 2021;114:106568. https://doi.org/10.1016/j.chb.2020.106568.
[10] Kuderer NM, Choueiri TK, Shah DP, Shyr Y, Rubinstein SM, Rivera DR, et al. Clinical impact of COVID-19 on patients with cancer (CCC19): a cohort study. The Lancet 2020;395:1907-18. https://doi.org/10.1016/S01406736(20)31187-9.

[11] Rivera DR, Peters S, Panagiotou OA, Shah DP, Kuderer NM, Hsu C-Y, et al. Utilization of COVID-19 Treatments and Clinical Outcomes among Patients with Cancer: A COVID19 and Cancer Consortium (CCC19) Cohort Study. Cancer Discov 2020;10:1514-27. https://doi.org/10.1158/21598290.CD-20-0941.

[12] Rubinstein SM, Steinharter JA, Warner J, Rini BI, Peters S, Choueiri TK. The COVID-19 and Cancer Consortium: A Collaborative Effort to Understand the Effects of COVID19 on Patients with Cancer. Cancer Cell 2020;37:738-41. https://doi.org/10.1016/j.ccell.2020.04.018.

[13] Poortmans PM, Guarneri V, Cardoso M-J. Cancer and COVID-19: what do we really know? The Lancet 2020;395:1884-5. https://doi.org/10.1016/S0140-6736(20) 31240-X.

[14] Covid in the U.S.: Latest Map and Case Count - The New York Times n.d. https://www.nytimes.com/interactive/ 2020/us/coronavirus-us-cases.html (accessed November 23, 2020).

[15] Omer SB, Malani P, del Rio C. The COVID-19 Pandemic in the US: A Clinical Update. JAMA 2020. https://doi.org/10.1001/jama.2020.5788.

[16] Kouzy R, Abi Jaoude J, Kraitem A, El Alam MB, Karam B, Adib E, et al. Coronavirus Goes Viral: Quantifying the COVID-19 Misinformation Epidemic on Twitter. Cureus n.d.;12. https://doi.org/10.7759/cureus.7255.

[17] Singh L, Bansal S, Bode L, Budak C, Chi G, Kawintiranon $\mathrm{K}$, et al. A first look at COVID-19 information and misinformation sharing on Twitter 2020.

[18] Sharma K, Seo S, Meng C, Rambhatla S, Liu Y. COVID19 on Social Media: Analyzing Misinformation in Twitter Conversations 2020.

[19] Mohammadi E, Thelwall M, Kwasny M, Holmes KL. Academic information on Twitter: A user survey. PLOS ONE 2018;13:e0197265. https://doi.org/10.1371/ journal.pone.0197265.

[20] Lovejoy K, Waters RD, Saxton GD. Engaging stakeholders through Twitter: How nonprofit organizations are getting more out of 140 characters or less. Public Relations Review 2012;38:313-8. https://doi.org/10. 1016/j.pubrev.2012.01.005.

[21] Tomlins SA, Laxman B, Varambally S, Cao X, Yu J, Helgeson BE, et al. Role of the TMPRSS2-ERG Gene Fusion in Prostate Cancer. Neoplasia 2008;10:177-IN9. https://doi.org/10.1593/neo.07822.

[22] Perner S, Demichelis F, Beroukhim R, Schmidt FH, Mosquera J-M, Setlur S, et al. TMPRSS2:ERG FusionAssociated Deletions Provide Insight into the Heterogeneity of Prostate Cancer. Cancer Res 2006;66:8337-41. https://doi.org/10.1158/0008-5472.CAN-06-1482.

[23] Mollica V, Rizzo A, Massari F. The pivotal role of TMPRSS2 in coronavirus disease 2019 and prostate cancer. Future Oncology 2020;16:2029-33. https://doi.org/ 10.2217/fon-2020-0571.

[24] Cumulative Cases - Johns Hopkins Coronavirus Resource Center n.d. https://coronavirus.jhu.edu/data/cumulativecases (accessed November 24, 2020). 